\title{
APROXIMACIÓN A LOS CONOCIMIENTOS QUE TIENE EL ALUMNADO AL FINALIZAR COU ACERCA DE LOS MATERIALES GEOLÓGICOS
}

VILA SANZ, F.' y ALBEROLA GINER, C. ${ }^{2}$

${ }^{1}$ Instituto de Bachillerato. Av. Campanar. Valencia.

${ }^{2}$ Instituto de Formación Profesional. Quart de Poblet. Valencia.

\begin{abstract}
SUMMARY
The answers given by 200 Geology students to a set of Multiple Choice questions on geological materials (both: rocks and minerals) are presented. The result of this test shows that there are some relevant aspects that remain unsufficiently known by the students after finishing COU. The necessity of reducing the syllabuses to avoid this fact is pointed out.
\end{abstract}

\section{INTRODUCCIÓN}

La preocupación que en los últimos tiempos puede observarse respecto a los distintos caminos posibles para mejorar la calidad de la enseñanza de las Ciencias Experimentales ha tenido -como ya es conocido- su reflejo mínimo en Geología (Mata y Anta 1986); quizás por ser la asignatura más minoritaria dentro del bloque đe experimentales. Sea cual sea la vía de investigación elegida, detectar y trabajar los preconceptos del alumna* do, enseñar una ciencia lo más incardinada posible en la vida cotidiana... presenta (de forma explícita o no) un đenominador común: la necesidad de cambiar los temarios que actualmente están en vigor. Sobre todo cuando los temas de investigación se centran en el nivel de COU, los cambios de currículos que se proponen conllevan siempre (aunque no sólo eso) la reducción en la extensión de los programas, que resultan enciclopédicos (Granda 1988), y superan en ocasiones en número y extensión los temas que se imparten en Geología general durante los primeros cursos universitarios (Rebollo 1990).

Uno de los bloques centrales de prácticamente todos los programas de Geología general es aquél que tiene que ver con el estudio de los materiales geológicos, tanto minerales como rocas, que suele convertirse en el aspecto más memorístico, inconexo, aburrido y peor aprendido de la Geología. La cantidad de conocimientos que se pretende que conozca el alumnado al finalizar los actuales BUP y COU es tan elevada que -como pretendemos ilustrar con este trabajo- sólo se consigue que retengan algunos aspectos del mismo -no siempre los fundamentales-, quedando lagunas importantes en la formación básica, que podrían eliminarse con la reestructuración de programas y el cambio en la metodología utilizada.

\section{MATERIALES Y MÉTODOS}

Los datos que se presentan en este trabajo forman parte de otro más amplio en el que hemos experimentado una alternativa al examen de Selectividad que se suele reali* zar para Geología.

El examen alternativo está formado por dos ejercicios: uno con 50 preguntas de Elección Múltiple y otro con 4 preguntas Estructuradas de respuesta corta, que en conjunto cubren la mayor parte del programa que actualmente se cursa en Geología de COU. Del ejercicio objetivo hemos extraído, en vista de lo sorprendente de los resultados, algunos de los ítems relacionados con los materiales geológicos. En la tabla I aparece, junto al número de la cuestión y siguiendo la taxonomía de Bloom (1974), la habilidad que creemos necesaria para resolverla, reflejada con una inicial: «C» hace referencia a comprensión, «CC» a conocimientos, $y$ «A» a aplicación. 
Las cuestiones fueron contestadas por una muestra de 200 alumnas y alumnos al finalizar el COU, en el curso académico 1989-90, durante el cual habían estudiado (y en general aprobado) Geología como asignatura optativa. El alumnado pertenecía a 19 centros de la Comunidad Valenciana, todos excepto uno institutos de Bachillerato.

Dicho ejercicio no se tuvo en cuenta en sus calificaciones de COU. Se advirtió al alumnado que sólo tenía que señalar una respuesta en cada f́tem, y que se penalizaría el error en la respuesta, pero no habría penalización para las preguntas no contestadas.

\section{RESULTADOS}

Los resultados obtenidos para las cuestiones que comentamos a continuación, se presentan en la tabla I.

\section{Tabla I}

Porcentajes de respuestas obtenidas para los diferentes ítems. En negrita se señalan las respuestas correctas. Innto al número de respuesta aparece: $\varangle \mathrm{C} »=$ comprensión, $\langle\mathrm{CC} »=$ conocimientos $\mathrm{y} \ll \mathrm{A} »=$ aplicación.

\begin{tabular}{|lccccll|}
\hline N. de item & $a$ & $b$ & $c$ & $d$ & Blancos & Error \\
\hline $1 \mathrm{C}$ & 8 & 15 & 5 & 54,5 & 17,5 & 77 \\
$2 \mathrm{CC}$ & $\mathbf{5 7 , 5}$ & 9 & 2,5 & 1 & 30 & 12,5 \\
$3 \mathrm{C}$ & 10,5 & 13 & 12 & 37,5 & 28,5 & 60 \\
$4 \mathrm{CC}$ & 2,5 & 9 & $\mathbf{3 6 , 5}$ & 28,5 & 23,5 & 40 \\
$5 \mathrm{~A}$ & 20,5 & $\mathbf{3 3 , 5}$ & 19 & 11 & 16 & 50,5 \\
$6 \mathrm{CC}$ & $\mathbf{3 2 , 5}$ & 26 & 10,5 & 11 & 20 & 47,5 \\
$7 \mathrm{CC}$ & 16,5 & $\mathbf{2 3 , 5}$ & 8 & 30,5 & 21,5 & 55 \\
$8 \mathrm{C}$ & $\mathbf{8 , 5}$ & 15,5 & $\mathbf{5 5 , 5}$ & 4 & 16,5 & 28 \\
$9 \mathrm{C}$ & 10,5 & 33 & 21 & 14,5 & 21 & 48 \\
$10 \mathrm{CC}$ & 5,5 & $\mathbf{7 5}$ & 3 & 1 & 15,5 & 9,5 \\
$11 \mathrm{CC}$ & 10,5 & 14 & $\mathbf{5 8}$ & 6,5 & 11 & 31 \\
$12 \mathrm{CC}$ & 11 & $\mathbf{6 7}$ & 5,5 & 3 & 13,5 & 19,5 \\
\hline
\end{tabular}

1. La birrefringencia de los minerales es una propiedad física:

a) típica de los cuerpos ópticamente isótropos;

b) que sólo se observa si se ilumina el cristal con luz polarizada;

c) que no pueden presentar los cuerpos ópticamente isótropos; d) que consiste en que se da la reflexión de dos rayos de luz al iluminar algunos minerales.

Más de la mitad de la población elige para explicar la birrefringencia la opción que menciona la existencia de dos rayos de luz, aunque dicho distractor trate de reflexión en lugar de refracción, que sería lo correcto. Es evidente, pues, que aunque recuerdan haber tratado dicha propiedad física en el aula, el concepto no ha sido aprendido. Por otro lado, parece claro que los conceptos de isotropía y anisotropía óptica no se conocen.

La birrefringencia de los minerales es una propiedad óptica que, pese a su dificultad, viene tratada incluso en muchos libros de texto, de primero de BUP. Hace falta una revisión a fondo en la forma de presentarla al alumnado, ya que, incluso en los libros de texto, suele resultar difícil de comprender.

\section{Un cristal es holoédrico si tiene:}

a) el número máximo de elementos de simetría del sistema cristalino;

b) la mitad del número máximo de elementos de simetría del sistema;

c) un tercio del número máximo de elementos de simetría del sistema;

d) un cuarto del número máximo de elementos de simetría del sistema.

Trata de uno de los aspectos más temidos tanto por el alumnado como por el profesorado de la asignatura. A pesar de que el conceto de holedría es de los más básicos en Cristalografía, sólo el $57,5 \%$ contesta correctamente y, a excepción đe otra cuestión de Paleontología que no presentamos en este trabajo, es la que más en blanco dejan de entre las 50 preguntas que formaban el ejercicio de cuestiones objetivas experimentado.

3. Que la alteración del granito genere los conocidos paisajes en «caos de bolas» o berrocales, se explica por:

a) la expansión diferencial de los minerales que componen el granito;

b) porque el granito presenta dos sistemas de diaclasas que se cortan perpendicularmente;

c) la descamación típica del granito;

d) la hidrólisis del cuarzo que favorece la fragmentación de la roca.

Suponemos que los resultados son un reflejo del mayor hincapié que en general hacemos -para explicar la alteración del granito- en la caolinización de feldespatos, frente a los planos preferentes por los que se realiza la misma. El alumnado tiende a buscar la alteración del mineral y señala como buena la respuesta $D$ que es la única que trata de la alteración de minerales; la consecuencia es que el $37,5 \%$ indica que tiene Iugar la hidrólisis del cuarzo.

\section{Se habla de dolomitización en calizas cuando:}

a) Ia caliza contiene además de calcita cualquier otro mineral; 
b) la caliza se encuentra mezclada con areniscas;

c) la caliza contiene carbonato de calcio y carbonato de magnesio;

d) Ia caliza se encuentra mezclada con arcillas.

A pesar de lo común que es el proceso de dolomitización en las rocas de nuestro entorno, sólo es indicado correctamente por el $36,5 \%$ de la población. Como se ve en la tabla, un porcentaje elevado de la población confunde las dolomías con las margas.

\section{A medida que avanza la consolidación magmática, el magma residual: \\ a) disminuye de viscosidad; \\ b) se enriquece en sílice de forma relativa; \\ c) se va haciendo más básico; \\ d) se empobrece relativamente en súlice.}

En vista de los resultados obtenidos en el resto de preguntas, es sorprendente que no sea contestado correctamente por un porcentaje aun menor de la población ya que precisa de un conocimiento profundo de la consoliđaciốn magmática.

6. Una de las diferencias entre el granito y la sienita es:

a) Ia sienita en general no tiene cuarzo;

b) Ia textura;

c) el granito no tiene ortosa;

d) la sienita en general no tiene feldespatoides.

Hay que destacar de esta pregunta no sólo el bajo porcentaje de respuestas correctas, sino también la gran cantidad del alumnado que basa la diferencia entre granito y sienita en la textura o en la inexistencia de ortosa en el granito.

7. En cuál de las opciones se presenta una roca volcánica de composición similar al granito:
a) andesita,
b) riolita,
c) traquita,
d) basalto.

El hecho de que el $30 \%$ de la población señale de forma equivocada el basalto, creemos que es un reflejo de la forma en que suelen presentarse las rocas intrusivas: siempre se utiliza el granito como representante de las plutónicas, y el basalto como representante típico de las volcánicas, lo que al parecer origina una tendencia a contraponerlas en todos los aspectos.

A continuación comentamos conjuntamete dos ítems que tratan sobre «esquistosidad».

8. En cuál de las siguientes rocas no puede aparecer esquistosidad:
a) pizarras,
b) gneis,
c) basalto,
d) micacita.

9. En las rocas metamórficas:

a) no pueden encontrarse planos de estratificación;

b) en lugar de planos de estratificación, se encuentran de esquistosidad;

c) pueden encontrarse ambos tipos de planos;

d) no puede encontrarse ninguno de ellos.

El concepto de esquistosidad nos parece básico y el alumnado parece conocerlo aunque el $8,5 \%$ piense que las pizarras no la presentan.

Habria que hacer, sin embargo, un esfuerzo para relacionar las estructuras de los distintos tipos de rocas, ya que sólo el $21 \%$ contempla que puedan existir planos de estratificación en las rocas metamórficas.

Las cuestiones 10,11 y 12 son tres ítems de conocimientos que fueron contestados correctamente, como se observa en la tabla I, por porcentajes elevados de la población y cuyo texto añadimos a continuación.

10. Una masa grande de roca plutónica en contacto irregular con las rocas encajantes, recibe el nombre de:
a) Iopolito,
b) batolito,
c) Iacolito,
d) facolito.

11. Una serie metamórfica es el conjunto de rocas metamórficas:
a) de igual composición mineralógica;
b) de igual facies;
c) derivadas de una inicial;
d) de igual estructura.

12. El mármol es una roca metamórfica del metamorfismo de:
a) yeso,
b) caliza,
c) arenisca,
d) arcilla.

\section{CONCLUSIONES Y PROPUESTAS}

Aunque no somos ajenas al hecho de que cambiando Ia terminología utilizada en las preguntas se pueden variar los resuitados obtenidos (Palacios et al. 1989), creemos que los datos expuestos son lo suficientemente significativos como para servir de ayuda a las propuestas de remodelación de programas, y quizás para oriertar la práctica cotidiana de la enseñanza de los materiales geológicos.

Cuestiones tan básicas para una formación en Geología general como la gran resistencia del cuarzo o la presencia de ortosa en la composición del granito no parece que 
queden lo suficientemente bien asentadas tras haber cursado los programas vigentes de Geología en BUP y COU. Es necesario dedicar mayor atención a aquellos aspectos de la Geología que se consideran fundamenta. les, en detrimento de la variedad de contenidos de los temarios.

La reducción sería menos drástica si, como ya han propuesto algunos autores (Anguita et al. 1984), se evitara en lo posible la repetición de contenidos en los diferentes niveles en los que se estudia Geología en favor de una programación más coherente y escalonada a to largo de la etapa secundaria que nos ocupa.

\section{REFERENCIAS BIBLIOGRÁFICAS}

ANGUITA, F., JIMENO, G., MORENO, F., SÁNCHEZ, N., VILLANUEVA, A., 1984. La enseñanza de la Geología, de la escuela a la Universidad, en el futuro inmediato. $/ I I$ simposio sobre Enseñanza de la Geología, pp. 213-234. Barcelona.

GRANDA, A., 1988. Esquemas conceptuales previos de los alumnos en Geología, Enseñanza de las ciencias, Vol. 6 (3), pp. 239-243.

KLOPFER, L.E., 1974. Evaluacion de los aprendizajes en Ciencias, en Evaluación del aprendizaje. (Troquel: Buenos Aires).
Aunque, dado el tipo de trabajo que presentamos no podemos sacar conclusiones generales acerca de la metodología adecuada, algunos datos apuntan hacia la necesidad de cambios en los métodos de enseñanza: los resultados obtenidos respecto a la inexistencia de planos de estratificación en las rocas y otros, y también observando y relacionando estos aspectos en las salidas al campo.

La tendencia general debía ser hacia una enseñanza más comprensiva. Como se ve en la tabla 1 , en general las cuestiones que requieren, para ser contestadas, habilidades diferentes a la de conocimientos son contestadas correctamente por porcentajes menores de la población.

MATA, A. y ANTA, C., 1986. Las orientaciones actuales de la didáctica de las Ciencias Experimentales en España (1985), Enseñanza de las Ciencias, Vol. 4 (3), pp. 233-246.

PALACIOS, C., LÓPEZ, F., GARROTE, R. y MONTES, P., 1989. Procesos de la Ciencia y desarrollo cognitivo en Bachillerato, Enseñanza de las Ciencias, Vol. 7 (2), pp. $132-140$.

REBOLLO, M., 1990. La Geología en el COU: un intento de cambio conceptual y metodológico. Actas del VI Simposio de enseñanza de la Geología, pp. 60-74. Tenerife. 